\title{
TANGLES AND THE GROMOV INVARIANT
}

\author{
COLIN C. ADAMS
}

(Communicated by Frederick R. Cohen)

\begin{abstract}
Previous results about mutant knots are extended to show that the Gromov invariant of a knot or link is preserved when a chain of tangles which make up the knot or link is permuted.
\end{abstract}

This note is an observation on a result of Ruberman in [4]. In what follows, a link will always mean a knot or a link.

If $L$ is a link in a given regular projection, and $S$ a sphere which intersects the knot transversely in four points and intersects the projection plane in a circle, we call $S$ a Conway sphere and that part of $L$ contained in $S$ a tangle. We assume that the four strands of $L$ leaving $S$ do so in the four compass directions NE, SE, NW and SW. If we cut $S^{3}$ open along $S$ and rotate $S 180$ degrees about an axis through a diameter of $S$ which either runs north-south or east-west in the projection plane or runs perpendicular to the projection plane, and then reglue $S$, the resulting link $L^{\prime}$ is called a mutation of $L$. In [4], Ruberman proved that if $L^{\prime}$ is a mutation of $L$, then $S^{3}-L$ has the same Gromov invariant as $S^{3}-L^{\prime}$. (See [1] or Chapter 6 of [6] for an introduction to the Gromov invariant.) In particular, if $S^{3}-L$ is hyperbolic, then $S^{3}-L^{\prime}$ is also hyperbolic and the two manifolds have the same hyperbolic volume.

This demonstrates that the hyperbolic volume is not a complete invariant for hyperbolic knots. In this note, we prove the following extension of Ruberman's result:

Theorem 1. Let $L$ be a link as in Figure 1 and let $L^{\prime}$ be a link which resembles $L$ except that the tangles have been permuted. Then $S^{3}-L$ has the same Gromov invariant as $S^{3}-L^{\prime}$. In particular, if $S^{3}-L$ is hyperbolic, then $S^{3}-L^{\prime}$ is also hyperbolic and the two manifolds have the same hyperbolic volume.

Proof. Since any permutation of the tangles of $L$ can be obtained by successive exchanges of two adjacent tangles, it is enough to prove the theorem for such an exchange. Let $T_{1}$ and $T_{2}$ be the two adjacent tangles which are to be exchanged.

Received by the editors May 18, 1988 and , in revised form, October 19, 1988.

1980 Mathematics Subject Classification (1985 Revision). Primary 57M25.

Supported in part by NSF Grant-DMS-8711495. 


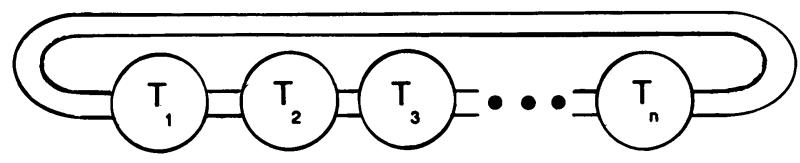

FIGURE 1

Treat $T_{1}$ and $T_{2}$ as a single tangle and flip it about a north-south axis in the projection plane. Then, if each of $T_{1}$ and $T_{2}$ are individually flipped about axes parallel to the first, the desired exchange is obtained. Thus, it suffices to prove that flipping any tangle preserves the Gromov invariant, and in the case $L$ is hyperbolic, it preserves hyperbolicity and the hyperbolic volume.

In the case that the Conway sphere bounding the tangle is incompressible and boundary-incompressible, this is exactly [4, Corollary 1.4 and Theorem 1.5]. In the case the Conway sphere boundary-compresses, it also must compress since the only possible orientable incompressible boundary-compressible surface in an orientable 3-manifold with all toral boundary components is an annulus (cf. [2, Lemma 3.4]). Hence we need only check the case when the Conway sphere compresses. Since $L$ is a knot or link, the boundary $\beta$ of the compressing disk must separate two of the punctures from the other two punctures on $S$. Let $S_{1}$ and $S_{2}$ be the two components of $S-\beta$. Then $S_{i}$ together with the compressing disk forms an incompressible annulus $A_{i}$ in the link complement, for $i=1,2$.

If $L$ is a prime nonsplittable link, as it must be in the hyperbolic case, each annulus is boundary-parallel, and the tangle is ambient isotopic to a rational tangle determined by the boundary curve $\beta$. The involution on $S$ commutes with all elements of the mapping class group and hence will preserve the curve $\beta$. Thus the rational tangle in $S$ will be sent back to itself under flipping and $L$ will be equivalent to $L^{\prime}$.

If $L$ is splittable, there could be additional components of $L$ trapped inside $A_{1}$ or $A_{2}$. However, since the Gromov invariant of a splittable link is simply the sum of the Gromov invariants for the nonsplittable sublinks which make it up, we can remove the extra unlinked components of $L$ and then replace them after the flipping operation without affecting the Gromov invariant. Similarly, since the Gromov invariant of a composite link is the sum of the Gromov invariants for the prime links which make it up, we can replace any knotting of arcs and link components inside $A_{1}$ and $A_{2}$ by trivial strands that the two annuli will be parallel to, flip the tangle and then replace the knotting, appropriately flipped. See [5] for these basic properties of the Gromov invariant. 
It follows from the proof of Theorem 1 that all of the additional results obtained in [4] extend to this situation. Thus, for example, if $K^{\prime}$ is a hyperbolic knot obtained by permuting a chain of tangles making up a knot $K$, the corresponding Dehn surgeries on $S^{3}-K$ and $S^{3}-K^{\prime}$ yield manifolds with the same Gromov invariant. Note that although an additional hypothesis that the mutation and the Conway sphere are unlinked is required in Theorem 5.5 of [4], no such hypothesis is required here, since the particular mutations utilized to permute the tangles will automatically be unlinked from the corresponding Conway spheres. Moreover, there is an obvious generalization of the above result to four-punctured spheres with incompressible punctures in any 3-manifold with toral boundary components and to tori with one or two incompressible punctures, orientable genus two surfaces and thrice-punctured spheres with incompressible punctures in 3-manifolds with toral boundary components, which are exactly the cases investigated in [4].

The author discovered the evidence for Theorem 1 using a computer program written by $\mathbf{J}$. Weeks, a description of which appears in [7]. It should be noted that two links related as in the hypotheses of Theorem 1 will have the same $P$ and $F$ polynomials (cf. [3]).

\section{REFERENCES}

1. M. Gromov, Volume and bounded cohomology, Publ. Math., Inst. Hautes Etud. Sci. 56 (1982), 5-100.

2. A. Hatcher, Torus decompositions, lecture notes by W. Floyd.

3. W. Lickorish and K. Millett, The new polynomial invariant of knots and links, Math Mag. 61, no. 1, Feb., 1988, 3-23.

4. D. Ruberman, Mutation and volumes of knots in $S^{3}$, Invent. Math. 90 (1987), 189-215.

5. T. Soma, The Gromov invariant of links, Invent. Math. 64 (1981), 445-454.

6. W. Thurston, The geometry and topology of 3-manifolds, notes, Princeton University, 1978.

7. J. Weeks, Hyperbolic structures on three-manifolds, Ph.D. dissertation, Princeton University, 1985.

Department of Mathematics, Williams College, Williamstown, Massachusetts 01267

Current address: Department of Mathematics, University of California, Santa Barbara, California 93106 